\title{
Spectral energy distribution of the metagalactic ionizing radiation field from QSO absorption spectra ${ }^{\star}$
}

\author{
I. I. Agafonova ${ }^{1}$, M. Centurión ${ }^{2}$, S. A. Levshakov ${ }^{1}$, and P. Molaro ${ }^{2}$ \\ 1 Department of Theoretical Astrophysics, Ioffe Physico-Technical Institute, 194021 St. Petersburg, Russia \\ e-mail: lev@astro.ioffe.rssi.ru \\ 2 Osservatorio Astronomico di Trieste, via G. B. Tiepolo 11, 34131 Trieste, Italy
}

Received 1 March 2005 / Accepted 30 May 2005

\begin{abstract}
A computational procedure is presented to estimate the spectral shape of the ionizing background between 1 and 10 Ryd by analyzing optically thin absorption systems in the spectra of high redshift quasars. The procedure is based on the response surface methodology from the theory of experimental design. The shape of the recovered UV background at $z \sim 3$ shows a significant intensity decrease between 3 and 4 Ryd compared to the metagalactic spectrum of Haardt \& Madau (1996, ApJ, 461, 20). This decrease is interpreted as produced by He II Gunn-Peterson effect. There are no features indicating a contribution from galaxies to the UV background which is therefore dominated by QSOs at $z \sim 3$.
\end{abstract}

Key words. cosmology: observations - line: formation - line: profiles - Galaxy: abundances galaxies: quasars: absorption lines

\section{Introduction}

The study of the spectral energy distribution (SED) in the radiation background resulting from flux emitted by all celestial sources is an important part of modern observational cosmology. The current paradigm assumes that the metagalactic ionizing background is formed by radiation of QSOs and galaxies (stars) reprocessed by the intergalactic medium (IGM). The SED evolves with cosmic time due to different contributions of galactic and QSO radiation, and to varying IGM opacity caused by the hydrogen and helium reionization.

Since the pioneering works of Chaffee et al. (1986) and Bergeron \& Stasinska (1986), the approach to recover the shape of the ionizing radiation field from the measurements of the intervening metal absorbers in QSO spectra has been employed in numerous studies. In particular, the energy range 1 Ryd $<E<10$ Ryd can be probed through the relative intensities of metal lines such as Si II-Si IV, C II-C IV, N III, N V, O VI. A common procedure consists of selecting a standard SED and checking whether it is consistent with measured column densities. This procedure can be appropriate for obtaining some general information about the SED, but its effectiveness and accuracy is rather low because of a lack of a search strategy.

In the present paper we describe a computational technique exploiting the response surface methodology from the theory of experimental design which enables a directed search for the shape of the ionizing background. The proposed method reliably recovers the main features in the spectral shape of the metagalactic flux by the analysis of optically thin QSO

* Based on observations obtained at the VLT Kueyen telescope (ESO, Paranal, Chile), the ESO programme 65.O-0474(A). absorption systems. Four absorption systems were selected to illustrate how this approach can be implemented in practice.

Three of these systems have redshifts $z \sim 3$. This redshift is of particular interest to study the traces of still not completely ionized He II (Reimers et al. 1997). In general, the presence of He II in the intergalactic medium affects the ionizing spectrum in the range $E>1$ Ryd due to He II Ly $\alpha$, two-photon reemission and to He II continuum absorption (Haardt \& Madau 1996; Fardal et al. 1998). The He II Ly $\alpha$ absorption troughs detected in spectra of five quasars (Vogel \& Reimers 1995; Zheng et al. 1998; Anderson et al. 1999; Heap et al. 2000; Smette et al. 2002; Jakobsen et al. 2003; Zheng et al. 2004a) may indicate the He II Gunn-Peterson effect (He II Ly $\alpha$ absorption in a diffuse IGM) at $z \sim 3$. Furthermore, recent FUSE observations of the He II Ly $\alpha$ forest towards HE 2347-4342 combined with observations of the H I Ly $\alpha$ forest at the VLT also revealed large scale variations in $\eta=\mathrm{He}$ II/H I which are still not well understood (Shull et al. 2004; Zheng et al. 2004b). One possible explanation is the presence of spatial fluctuations in the metagalactic ionizing field at $E \gtrsim 4$ Ryd. To confirm this suggestion, additional observations at different redshifts and on different sightlines are needed. However, direct measurements of He II Ly $\alpha$ absorption at $303.78 \AA$ are very problematic due to almost complete light blotting of distant QSOs by the intervening Lyman limit or damped Ly $\alpha$ systems (LLSs and DLAs, respectively). In this context, the proposed approach to estimate the shape of the ionizing background from metal absorption systems is of great importance.

The structure of the paper is as follows. The procedure to recover the shape of the underlying UV continuum and an example illustrating how to use it are described in Sect. 2. 
This section also contains a brief description of a computational method used to invert the observed line profiles - the Monte Carlo Inversion (MCI). The detailed analysis of absorption systems used for the SED estimations is given in Sect. 3. The results are discussed and summarized in Sect. 4. An example of an experimental design is given in the Appendix.

\section{Computational methods}

\subsection{Shape of the ionizing radiation}

Here we give a description of how the SED of the ionizing radiation can be estimated from metal line profiles observed in optically thin absorption systems. The method is based on the response surface methodology used in experimental design (see, e.g., Box et al. 1978, Chap. 15).

A basic UV spectrum is taken as an initial guess. The estimated column densities of different ions and a photoionization code (e.g., CLOUDY, Ferland 1997) are used to derive the ionization parameter, metallicity and element abundance ratios. If the observed column densities are well reproduced with this trial spectrum, it implies that the initial guess was appropriate. If, however, the observed column densities cannot be reproduced or/and some other inconsistencies arise, then the assumed shape of the UV background is to be adjusted.

To allow for quantitative estimations, the shape of the UV continuum must be parameterized. In general, the shape of the ionizing spectrum can be specified by a piecewise continuous function, e.g. by a set of power laws and/or exponents. In particular, in the range $1 \mathrm{Ryd}<E<10 \mathrm{Ryd}$ relevant for the ions frequently observed in QSO spectra, the following variables (called "factors" in the experimental design) can be used to describe the main features of the SED (Fig. 1):

A first region between 1 Ryd and the He I break is characterized by a slope $f_{1}$ (power law exponent) and by the position $f_{2}$ of the He I break. A second region can be defined between the He I and He II breaks (point $A$ ) with $f_{3}$ and $f_{4}$ the slope and the position of the He II break, respectively. Since the energy depression can be relevant, we introduce here also $f_{5}$ which defines the depth of the break $\left[f_{5}=\log \left(J_{\mathrm{B}} / J_{\mathrm{A}}\right)\right]$, and $f_{6}$ - the slope between points $A$ and $B . f_{7}$ characterizes the energy spectrum in the region $B-C$. To account for a possible bump around 3 Ryd due to recombinations within the clumpy intergalactic gas (He II Ly $\alpha$ emission, He II two-photon continuum emission and He II Balmer continuum emission), we introduce factors $f_{8}$ and $f_{9}$ which describe the amplitude of the bump and its width, respectively.

The energy of the far UV cut off (point $C$ ) when taken above 100 Ryd does not affect the fractional ionizations of ions we are interested in. In all computations described in subsequent sections this energy and the slope after it are kept fixed at 128 Ryd and -1.5 , respectively. Note that the positions of the He I and He II breaks can be shifted due to large scale motions and/or superposition of different spectra (e.g. stellar+metagalactic), and hence, in general the values of $f_{2}$ and $f_{4}$ may not be equal to 1.8 Ryd and 4 Ryd, respectively. Additional factors can be defined to describe some particular

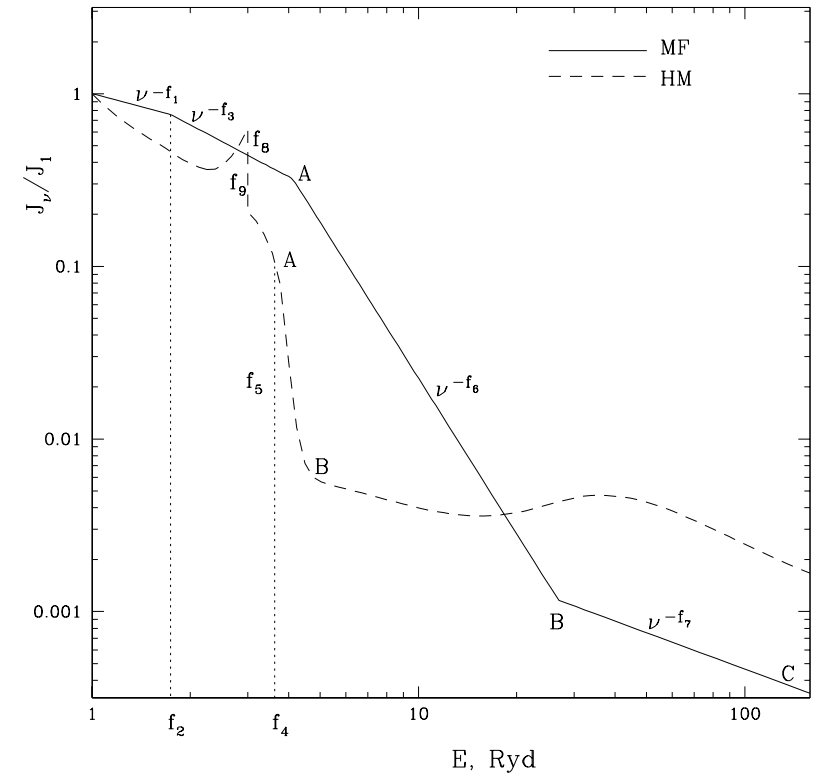

Fig. 1. Schematic picture of the $z=3$ metagalactic (dashed line) and AGN-type (smooth line) ionizing backgrounds from Haardt \& Madau (1996) and Mathews \& Ferland (1987), respectively. The spectrum is normalized so that $J_{v}(h v=1 \mathrm{Ryd})=1$. The emission bump at $3 \mathrm{Ryd}$ in the metagalactic spectrum is caused by reemission of He II Ly $\alpha$, He II two-photon continuum emission and He II Balmer continuum emission from intergalactic clouds. The definition of factors $\left\{f_{i}\right\}$ is given in Sect. 2.1.

features of the SED such as, for example, those caused by He II Ly $\alpha$ absorption in a diffuse IGM (see Sect. 3 below).

In this notation, an ionizing spectrum is determined as a point $\left\{f_{1}, \ldots, f_{k}\right\}$ in the $k$-dimensional factor space. The number and selection of factors depend on a particular spectral shape. For instance, the metagalactic spectrum at $z=3$ calculated by Haardt \& Madau (1996, hereafter HM) has coordinates $\left\{f_{1}, f_{4}, f_{5}, f_{6}, f_{7}, f_{8}, f_{9}\right\}=\{-1.4,0.55,-1.3,-13.0,-0.45$, $0.46,0.11\}$ with $f_{1}$ standing for the entire slope between 1 Ryd and $f_{4}$, whereas the point $\left\{f_{1}, f_{2}, f_{3}, f_{4}, f_{5}, f_{6}, f_{7}\right\}=\{-0.5,0.25$, $-1.0,0.61,-2.5,-3.0,-0.7\}$ corresponds to the AGN-type spectrum of Mathews \& Ferland (1986, hereafter MF) (all energies are given in $\log _{10}$ ).

The next step of the shape adjustment procedure is to find a direction in the factor space which leads to the UV background with more appropriate characteristics. The starting ("null") point is represented by the initial spectrum. A set of new trial spectra is produced by varying the factor values around the "null" point in accordance with a special scheme called "experimental (factorial) design". The experimental design is represented by a matrix with $n(n>k)$ rows containing particular values of factors (an example of the experimental design is given in Appendix).

To evaluate the fitness of each trial ionizing spectrum a numerical measure $\tilde{\mathcal{R}}$ (usually called "response") is to be defined in such a way that larger values of $\tilde{\mathcal{R}}$ correspond to increasing fitness. In general, the choice of $\tilde{\mathcal{R}}$ occurs rather heuristically and accounts for the information obtained with the initial UV spectrum and for any a priori information (like, e.g., allowable 
element abundance ratios). For illustration consider the following two examples.

Assume that a set of silicon ( $\mathrm{Si}$ II, Si III, Si IV) and carbon (C II, C III, C IV) lines is observed in an absorption system, and that the initial UV spectrum overestimates the column densities of Si II and C IV and underestimates those of Si IV and C II. Then the response can be written as

$\tilde{\mathcal{R}}=\frac{\mathrm{C} \text { II }}{\mathrm{CIV}} \times \frac{\mathrm{Si} I V}{\mathrm{Si} I \mathrm{II}}$,

i.e., the search should go towards increasing the product of these ratios.

Another example concerns an absorption system with the observed lines of Si III, Si IV, and C IV. The ratio Si III/Si IV determines the ionization parameter $U$. Using the corresponding ion fractions $\Upsilon_{i}$ the ratio

$$
\frac{\mathrm{Si}}{\mathrm{C}}=\frac{\mathrm{SiIV}}{\mathrm{CIV}_{\mathrm{IV}}} \frac{\Upsilon_{\mathrm{CIV}}}{\Upsilon_{\mathrm{SiIV}}}
$$

can be calculated. Both observations and theoretical considerations give for this ratio a safe upper limit $\mathrm{Si} / \mathrm{C}<3(\mathrm{Si} / \mathrm{C})_{\odot}$. If, for instance, the initial UV background delivers $\mathrm{Si} / \mathrm{C}=$ $10(\mathrm{Si} / \mathrm{C})_{\odot}$, then the search for a new SED can be governed by the ratio

$\tilde{\mathcal{R}}=\frac{\Upsilon_{\mathrm{SiIV}}}{\Upsilon_{\mathrm{CIV}}}$,

calculated for the ionization parameter $U$ given by the observed column density ratio Si III/Si IV (note that the value of $U$ depends on the shape of the trial spectrum). More examples are presented in the subsequent sections.

The calculated responses $\left\{\tilde{\mathcal{R}}_{i}\right\}_{i=1}^{n}$ can be considered as points belonging to some surface in $k$-dimensional factor space (referred to as "response surface"). To determine the direction of steepest ascent the response surface should be described by some analytical function. A standard linear model is a reasonable first approximation:

$\mathcal{R}=\sum_{i=1}^{k} \alpha_{i} \hat{f}_{i}+\beta$

where $\left\{\alpha_{i}\right\}_{i=1}^{k}$ are factor effects, $\hat{f}_{i}$ is the scaled and centered value of the $i$ th factor, $\hat{f}_{i}=\left(f_{i}-f_{0, i}\right) / s_{i}, s_{i}$ is some suitable scale (range of variation) of the $i$ th factor, and $\beta=\alpha_{0}+\sum_{i, j=1}^{k} \alpha_{i, j} \hat{f}_{i} \hat{f}_{j}$ stands for the joint effect of the free term $\alpha_{0}$ and of the nonlinear term that contains also the interaction of factors.

The coefficients $\alpha_{i}$ and $\beta$ and their dispersions are calculated from $n$ values of $\mathcal{R}$ according to special formulae (depending on the type of experimental design used). The validity of the planar data model (1) can be checked by comparison of the estimation, $\beta^{\prime}$, with the value of $\mathcal{R}_{0}$ at the "null point", i.e., when $f_{i}=f_{0, i}$. The statistical significance of the difference $\left(\mathcal{R}_{0}-\beta^{\prime}\right)$ points to non-negligible non-linear effects. In this case the higher-order data model is to be used with corresponding higher-order experimental design.

Given the factor effects $\left\{\alpha_{i}\right\}$, the maximization of $\mathcal{R}$ is obtained by moving the factor values from the "null" point in the direction normal to the response surface. This movement is performed stepwise until either the desired UV background is found (i.e. the one which reproduces self-consistently all observed column densities) or the validity limit of the employed data model is reached. In the latter case the adjustment procedure should be repeated, this time with a newly obtained UV spectrum as a "null" point.

The initial set of factors may turn out to be redundant, i.e. some of the factor effects $\alpha_{i}$ may be statistically insignificant. This means that the corresponding features in the spectral shape do not affect the absorption line profiles included in the analysis. Such insignificant factors are removed from the data model (1) and are fixed at some appropriate level. The effects of the remaining $m$ factors are then recalculated using the corresponding $m$-factorial design.

The uncertainty of the recovered spectral shape requires some comments. The spectrum of the ionizing radiation, $J_{v}$, defines the rates $\left(\mathrm{s}^{-1}\right)$ of the photoionization processes, $\Gamma_{i j}$, through the integral

$\Gamma_{i j}=\int_{v_{i j}}^{\infty} \frac{4 \pi J_{v}}{h v} \sigma_{i j}(v) \mathrm{d} v$

where $\sigma_{i j}(v)$ is the photoionization cross section at frequency $v$ for species $i$ in ionization state $j$, and $v_{i j}$ is the threshold frequency for photoionization.

Thus, the SED is obtained by solving the integral equation which is a classic ill-posed problem. Its solution depends crucially on the number of constraints included in the analysis. When applied to our problem, this means that the SED is best recovered if the corresponding absorption system reveals many lines of different metals in different ionization stages. If only a few metal lines are observed, they can be used to recover the shape not in the entire range $1 \mathrm{Ryd}<E<10 \mathrm{Ryd}$, but in some narrower regions. For example, the lines of $\mathrm{C}$ III, C IV and O VI allow us to estimate the SED in the vicinity of the He II break (3 Ryd $<E<4.5$ Ryd).

\subsection{The Monte Carlo Inversion procedure}

Absorption systems are analyzed by means of the Monte Carlo Inversion (MCI) procedure described in detail in Levshakov et al. (2000, hereafter LAK), and with modifications in Levshakov et al. $(2002,2003 a, b)$. Here we briefly outline the basics needed to understand the results presented below in Sect. 3.

The MCI is based on the assumption that all lines observed in the absorption system are formed in a continuous medium where the gas density, $n_{\mathrm{H}}(x)$, and velocity, $v(x)$, fluctuate from point to point giving rise to complex profiles (here $x$ is the space coordinate along the line of sight).

The MCI also assumes that within the absorber the metal abundances are constant, the gas is optically thin for the ionizing UV radiation, and the gas is in thermal and ionization equilibrium. The intensity and the spectral shape of the background ionizing radiation are treated as external parameters.

The radial velocity $v(x)$ and gas density $n_{\mathrm{H}}(x)$ are considered as two continuous random functions which are represented by their sampled values at equally spaced intervals $\Delta x$. 


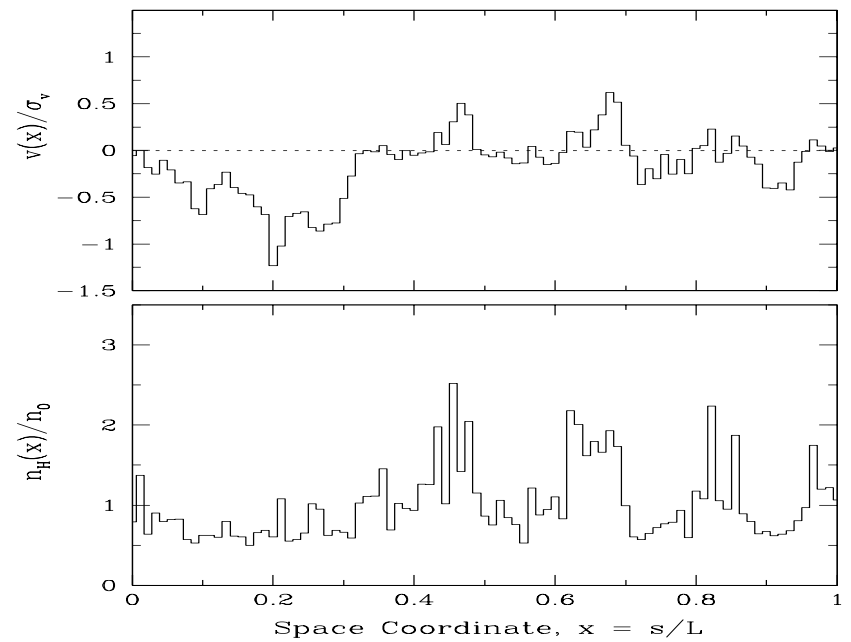

Fig. 2. Velocity (upper panel) and gas density (lower panel) distributions along the line of sight used to produce the mock absorption spectra which are shown in Fig. 4.

The computational procedure uses the adaptive simulated annealing. The fractional ionizations of all elements included in the analysis are computed at each space coordinate $x$ with the photoionization code CLOUDY.

In the MCI procedure the following physical parameters are directly estimated: the mean ionization parameter $U_{0}$, the total hydrogen column density $N_{\mathrm{H}}$, the line-of-sight velocity dispersion $\sigma_{\mathrm{v}}$, and density dispersion $\sigma_{\mathrm{y}}$, of the bulk material $\left[y \equiv n_{\mathrm{H}}(x) / n_{0}\right]$, and the chemical abundances $Z_{\mathrm{a}}$ of all elements involved in the analysis. With these parameters we can further calculate the column densities $N_{\mathrm{a}}$ for different species, and the mean kinetic temperature $T_{\text {kin }}$. If the absolute intensity of the UV background is known, then the mean gas number density $n_{0}$, and the line-of-sight thickness $L$ of the absorber can be evaluated as well.

In general, the uncertainties of the fitting parameters $U_{0}$, $N_{\mathrm{H}}, \sigma_{v}, \sigma_{y}$, and $Z_{a}$ are about $15 \%-20 \%$ (for data with $S / N \gtrsim$ 30 ) and the errors of the estimated column densities are less than $10 \%$. However, in individual absorption systems, the accuracy of recovered values can be lower due to different reasons such as partial blending of line profiles, saturation of profiles or absence of lines of subsequent ionic transitions.

The procedure of spectral shape adjustment described in the preceding section is used in a modified form. From a computational point of view, it is more convenient within the MCI to evaluate responses $\tilde{\mathcal{R}}$ using ion fractions rather then column densities (at a given metallicity, the column density of an ion is proportional to its fraction). The procedure stops when the spectral shape ensuring $\chi^{2} \lesssim 1$ for all lines observed in the system is found.

\subsection{Testing the SED recovery procedure}

In order to test the procedure of the SED adjustment we prepared a mock absorption line spectrum using the density and velocity distributions plotted in Fig. 2, the UV ionizing background shown in Fig. 3 (solid line) and the model parameters

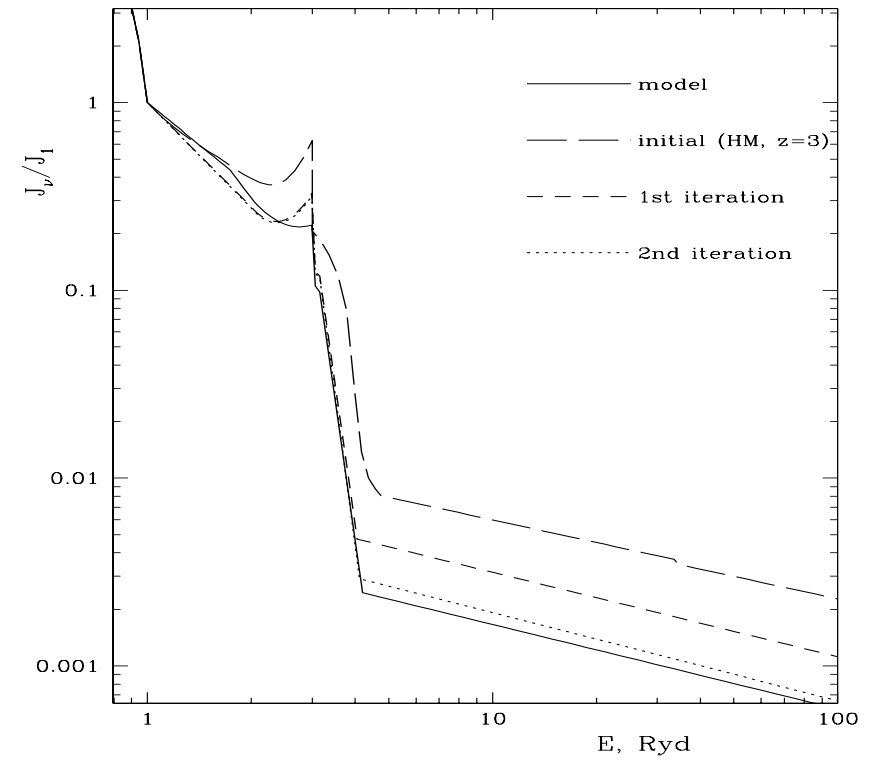

Fig. 3. A model ionizing background (smooth line) used to calculate the mock spectra shown in Fig. 4. As an initial approximation for the restoring procedure an HM-type spectrum is taken (long-dashed line). The results of the first and second iterations are illustrated by the shortdashed and dotted lines, respectively.

listed in Table 1, Col. 2. The resulting absorption lines after convolution of the intensities with a Gaussian-type pointspread function of $F W H M=7 \mathrm{~km} \mathrm{~s}^{-1}$ and the addition of white noise with a dispersion of $0.02(S / N=50)$ are shown in Fig. 4 by points with error bars. The chosen UV background can be produced by a mixture of stellar and metagalactic spectra (see, e.g., Giroux \& Shull 1997).

The procedure requires an input SED. As an initial guess for the underlying UV background we took the metagalactic spectrum of HM shown in Fig. 3 by the long-dashed line. This spectrum was defined by 7 factors $\left\{f_{1}, f_{4}, f_{5}, f_{6}, f_{7}, f_{8}, f_{9}\right\}$ with the meanings described in Sect. 2.1. The only change is for $f_{1}$ which now stands for the entire slope between 1 Ryd and the He II break at $f_{4}$.

The best MCI solution gives the physical parameters listed in Table 1, Col. 3. The corresponding synthetic absorptions are shown in Fig. 4 by the dotted lines. As expected, not all absorptions are well reproduced, since the UV background used differs from the one used to generate the mock lines. An underestimation of the C III and Si III intensities along with overestimation of C IV is clearly seen in Fig. 4.

To calculate responses, the following expression was chosen:

$\tilde{\mathcal{R}}=\log \left(\frac{\Upsilon_{\mathrm{CIII}}}{\Upsilon_{\mathrm{CIV}}} \frac{\Upsilon_{\mathrm{Si} \text { III }}}{\Upsilon_{\mathrm{SiII}}}\right)$,

with an additional constraint $\log \left(\Upsilon_{\text {Si III }} / \Upsilon_{\text {Si IV }}\right) \gtrsim 0.4$ [i.e. the fractions of the corresponding ions are calculated for the value of $U$ determined by the condition $\left.\log \left(\Upsilon_{\text {Si III }} / \Upsilon_{\text {Si IV }}\right) \gtrsim 0.4\right]$.

Factors were varied at 2 levels according to a 7-factorial saturated simplex design which gave 8 new trial UV spectra (see Appendix). These spectra were inserted into CLOUDY and with the obtained fractional ionizations the responses for 
Table 1. Physical parameters for model absorption system shown in Fig. 4.

\begin{tabular}{|c|c|c|c|c|}
\hline $\begin{array}{l}\text { Parameter } \\
\text { (1) }\end{array}$ & $\begin{array}{l}\text { Model } \\
\text { values } \\
(2)\end{array}$ & $\begin{array}{l}\text { Estimated with } \\
\text { initial SED } \\
\text { (3) }\end{array}$ & $\begin{array}{c}\text { Estimated with } \\
\text { 1st iteration SED } \\
\text { (4) }\end{array}$ & $\begin{array}{c}\text { Estimated with } \\
\text { 2nd iteration SED } \\
\text { (5) }\end{array}$ \\
\hline$U$ & $1.55 \mathrm{E}-2$ & $5.7 \mathrm{E}-2$ & $1.3 \mathrm{E}-2$ & $1.4 \mathrm{E}-2$ \\
\hline$N_{\mathrm{H}}, \mathrm{cm}^{-2}$ & 1.2E19 & $2.5 \mathrm{E} 18$ & 8.4E18 & $1.0 \mathrm{E} 19$ \\
\hline$\sigma_{\mathrm{v}}, \mathrm{km} \mathrm{s}^{-1}$ & 20.0 & 17.1 & 22.2 & 18.0 \\
\hline$\sigma_{\mathrm{y}}$ & 0.40 & 0.56 & 0.50 & 0.48 \\
\hline$Z_{\mathrm{C}}$ & $8.0 \mathrm{E}-6$ & $2.7 \mathrm{E}-5$ & $1.0 \mathrm{E}-5$ & $9.0 \mathrm{E}-6$ \\
\hline$Z_{\mathrm{Si}}$ & $1.0 \mathrm{E}-6$ & $3.8 \mathrm{E}-6$ & $1.6 \mathrm{E}-6$ & $1.2 \mathrm{E}-6$ \\
\hline$N(\mathrm{H} \mathrm{I}), \mathrm{cm}^{-2}$ & 3.4E15 & 3.7E15 & 3.4E15 & $3.4 \mathrm{E} 15$ \\
\hline$N(\mathrm{C}$ II $), \mathrm{cm}^{-2}$ & $2.5 \mathrm{E} 12$ & 3.0E12 & $2.9 \mathrm{E} 12$ & $2.6 \mathrm{E} 12$ \\
\hline$N(\mathrm{C}$ III $), \mathrm{cm}^{-2}$ & 8.1E13 & $5.5 \mathrm{E} 13$ & $6.9 \mathrm{E} 13$ & 7.7E13 \\
\hline$N(\mathrm{C}$ IV $), \mathrm{cm}^{-2}$ & $9.0 \mathrm{E} 12$ & $9.9 \mathrm{E} 12$ & $9.2 \mathrm{E} 12$ & $8.6 \mathrm{E} 12$ \\
\hline$N(\mathrm{Si}$ II $), \mathrm{cm}^{-2}$ & $3.5 \mathrm{E} 11$ & 4.2E11 & 3.9E11 & $3.5 \mathrm{E} 11$ \\
\hline$N(\mathrm{Si}$ III $), \mathrm{cm}^{-2}$ & $5.5 \mathrm{E} 12$ & $5.2 \mathrm{E} 12$ & $5.4 \mathrm{E} 12$ & $5.3 \mathrm{E} 12$ \\
\hline$N(\mathrm{Si}$ IV $), \mathrm{cm}^{-2}$ & $1.9 \mathrm{E} 12$ & $2.2 \mathrm{E} 12$ & $1.8 \mathrm{E} 12$ & $1.9 \mathrm{E} 12$ \\
\hline
\end{tabular}

Note: $Z_{\mathrm{X}}=N_{\mathrm{X}} / N_{\mathrm{H}}$.

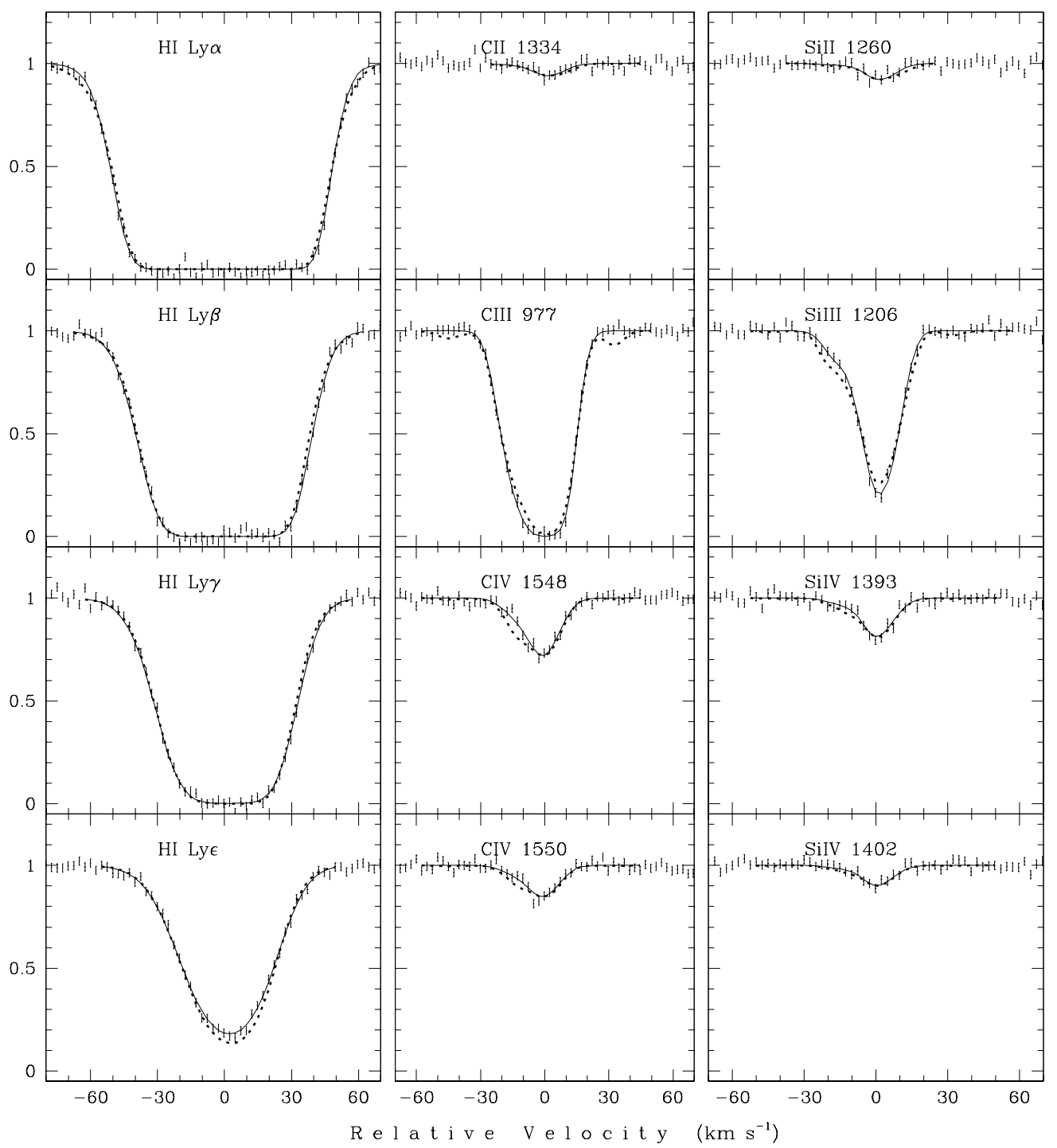

Fig. 4. Mock spectra of hydrogen and metal absorption lines (dots with $1 \sigma$ error bars). The MCI solution corresponding to the initial ionizing spectrum is shown by the dotted curves. The smooth curves are the synthetic profiles obtained with recovered 2nd iteration SED (dotted line in Fig. 3). 
each spectrum were evaluated. The factor effects were then estimated, the linearity of the data model checked, and a new UV background produced by moving the factor values in the direction normal to the response surface.

The new solution for the background is shown in Fig. 3 by the short-dashed line, and the results of the MCI calculations are given in Table 1, Col. 4. The fit of synthetic profiles to most absorption lines becomes much better, but C III, C IV and Si IV lines still have large $\chi^{2}$ values. Thus, the adjustment procedure was repeated once more, with the same set of factors, but different response

$\tilde{\mathcal{R}}=\log \left(\frac{\Upsilon_{\mathrm{CIV}}}{\Upsilon_{\mathrm{CII}}} \frac{\Upsilon_{\mathrm{SiIV}}}{\Upsilon_{\mathrm{SiI}}}\right)$,

with constraints $\log \left(\Upsilon_{\mathrm{CHII}} / \Upsilon_{\mathrm{CIV}}\right) \quad>\quad 0.9$ and $\log \left(\Upsilon_{\text {Si III }} / \Upsilon_{\text {Si IV }}\right)>0.5$.

The resulting UV spectrum is shown in Fig. 3 by dots, and the corresponding synthetic profiles by the smooth lines in Fig. 4. The recovered parameters are listed in Col. 5, Table 1. Now all absorption lines are well reproduced, and, thus, the inverse procedure can be considered completed.

This test demonstrates both the effectiveness of the adjustment procedure and its limitations. Starting from a standard spectral shape, we were able to recover correctly all significant features of the underlying ionizing spectrum such as the depth of the He II break and its shift to the lower energies. On the other hand, some fine features of the underlying spectrum (the break at $1.8 \mathrm{Ryd}$, the small bump at $3 \mathrm{Ryd}$ ) cannot be reproduced with the adopted noise level. Higher $S / N$ data would be more appropriate for this case.

\section{Application to observed absorption systems}

Here we analyze some QSO absorption systems using the procedure described in the preceding section. All computations below were performed with laboratory wavelengths and oscillator strengths taken from Morton (2003). Solar abundances were taken from Asplund et al. (2005).

\subsection{Absorption system at $\mathrm{z}_{\mathrm{abs}}=2.9171$ towards HE 0940-1050}

This absorption system, shown in Fig. 5, exhibits higher order HI Lyman series lines and transitions of C II/C III/C IV and Si II/Si III/Si IV.

The system was described in detail in Levshakov et al. (2003c) as an extremely low metallicity LLS with $N(\mathrm{HI})=3 \times$ $10^{17} \mathrm{~cm}^{-2}$ and $Z=0.001 Z_{\odot}$. Levshakov et al. also noted that the observed line intensities were inconsistent with the HM metagalactic ionizing spectrum, and that better fitting of the observed profiles corresponded to the ionizing spectrum having a significantly enhanced He II re-emission bump at 3 Ryd. This result was obtained by a "trial and error" method. Here we re-analyze the $z_{\mathrm{abs}}=2.9171$ system using a directed search.

With the HM ionizing spectrum at $z=3$ as an initial guess we obtained the synthetic profiles shown in Fig. 5 by dotted curves. Strong overestimation of Si II $\lambda 1260 \AA$ is clearly seen along with underestimation of C II $\lambda 1334 \AA$ and Si IV $\lambda 1393 \AA$. Whether the C III $\lambda 977 \AA$ line is underestimated or not cannot be decided unambiguously at this stage of investigation since the apparent intensity may be due to contamination by a Ly $\alpha$ interloper. The HM spectrum was parameterized using the same 7 factors as in the example described in Sect. 2.3. The responses were calculated in the form

$\tilde{\mathcal{R}}=\log \left(\frac{\Upsilon_{\mathrm{CII}}}{\Upsilon_{\mathrm{CIV}}} \frac{\Upsilon_{\mathrm{SiIV}}}{\Upsilon_{\mathrm{Si} I \mathrm{II}}}\right)$.

The design employed was the 7-factorial saturated simplex design (see Appendix). The first-iteration ionizing spectrum revealed a sharp He II break shifted to 3 Ryd and the He II re-emission bump significantly increased as compared to the initial approximation (Fig. 6, dotted line). This spectrum provided better fitting to the observed profiles (e.g., all carbon lines including $\mathrm{C}$ III were now described with $\chi^{2} \sim 1$ ), but the profiles of Si II and Si IV remained, correspondingly, over- and underestimated.

The next iteration of the SED adjustment was performed with the same set of factors, with the design and response augmented with constraints $\log \left(\Upsilon_{\mathrm{C} \text { III }} / \Upsilon_{\mathrm{CIV}}\right)<1$ and $\log \left(\Upsilon_{\text {Si III }} / \Upsilon_{\text {Si IV }}\right)>0.1$ to enable a self-consistent description of the C III and Si III lines. However, this step failed to produce any improvement. Attempts to include additional factors describing the He I break (see Fig. 1) or to employ other forms of the responses failed as well.

The solution was found after performing model calculations (using CLOUDY) of radiation transmission through a plane-parallel absorbing cloud. For an HM-type ionizing spectrum, $\eta=N(\mathrm{He}$ II $) / N(\mathrm{HI})>50$ and the absorber with $N(\mathrm{H} \mathrm{I})=3 \times 10^{17} \mathrm{~cm}^{-2}$, marginally optically thick in the hydrogen continuum, is certainly opaque in He II. The radiation in the He II continuum is effectively absorbed by such a cloud and a part of this radiation is reemitted as the He II $\lambda 304 \AA$ Ly $\alpha$ and two-photon reemission, and He II Balmer continuum emission. As a result, the incident HM spectrum being transmitted through the absorber with $N(\mathrm{HI})=3 \times 10^{17} \mathrm{~cm}^{-2}$ reveals a sharp and deep break at 4 Ryd and a strong emission line at 3 Ryd of about twice the incident intensity. Thus, the increased intensity at $E \lesssim 3 \mathrm{Ryd}$ in the ionizing spectrum recovered from the $z_{\text {abs }}=2.92$ absorber is probably due to auto-emission of the cloud.

However, in the recovered spectrum a strong intensity depression starts just above $3 \mathrm{Ryd}$, and this result has been reproduced in all trials with different responses and different sets of factors. At the same time, the model calculations show that the spectral range 3 Ryd $<E<4$ Ryd remains unaffected by the processes inside the cloud, i.e., if the intensity depression in this range is not present in the incident spectrum, then it does not appear in the transmitted one. This means that the depression at $E \gtrsim 3$ Ryd in the ionizing spectrum is produced by the processes outside the cloud. We can assume that this is a manifestation of He II Ly $\alpha \lambda 304 \AA$ absorption arising in both smoothly distributed IGM (He II Gunn-Peterson effect) and discrete Ly $\alpha$ forest clouds (line blanketing). He II Ly $\alpha$ absorption in the diffuse IGM gas results in the absorption trough blueward of the resonant wavelength $304 \AA$ (3 Ryd). This trough 


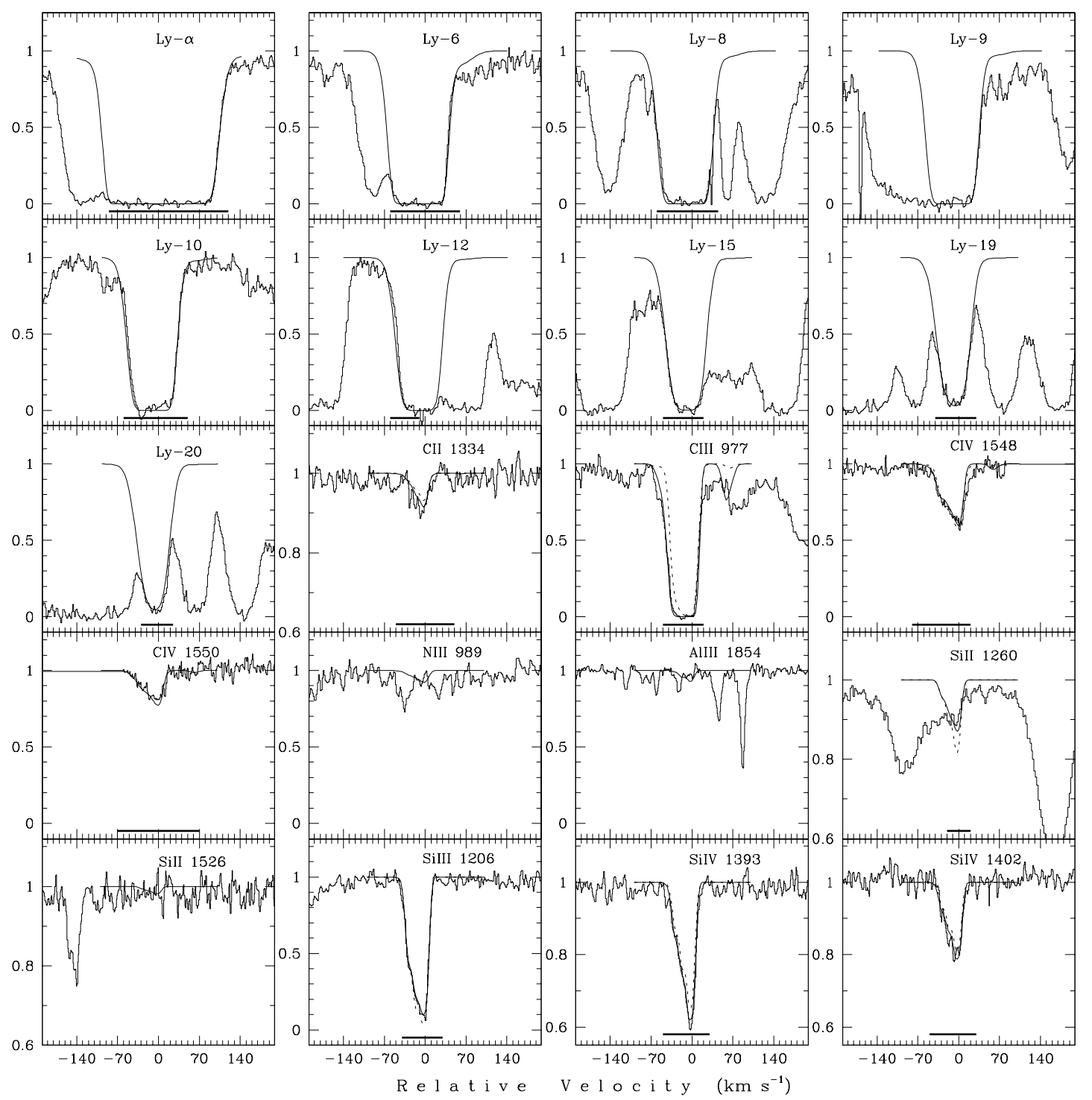

Fig. 5. Hydrogen and metal absorption lines associated with the $z_{\mathrm{abs}}=2.9171$ system towards HE 0940-1050 (histograms). Synthetic profiles corresponding to the MCI solution with the $z=3 \mathrm{HM}$ ionizing spectrum (smooth line in Fig. 6) are shown by the dotted curves, whereas those calculated with the final adjusted SED (short-dashed line in Fig. 6) are the smooth curves. The corresponding physical parameters are listed in Table 2, Col. 2. Bold horizontal lines mark pixels included in the optimization procedure. The zero radial velocity is fixed at $z=2.9171$.

together with the subsequent absorption in the He II continuum ( $E \geq 4$ Ryd) produces a winding structure in the spectral shape at $E \gtrsim 3$ Ryd. As a first approximation, this structure can be described as a straight step (see Fig. 6).

After this consideration, a new set of factors was defined to account for this step-like form (Fig. 6): $f_{1}$ - the slope between 1 and 3 Ryd; $f_{2}$ - the depth of the He II absorption through; $f_{3}-$ the energy of the He II ionization break; $f_{4}-$ the depth of the He II ionization break; $f_{5}$ - the slope of the He II ionization break; $f_{6}, f_{7}-$ the height and width of the He II re-emission bump; $f_{8}-$ the slope after the He II ionization break. In the following analysis, factors $f_{3}$ and $f_{8}$ turned out to have low effects and were set to 4 Ryd and -0.45 , respectively.

The final UV background is shown by the short-dashed line in Fig. 6. The synthetic line profiles calculated with this SED are plotted by the smooth solid lines in Fig. 5 and the corresponding physical parameters are listed in Table 2, Col. 2.
The predicted column density of He II for this absorber is $2.8 \times 10^{19} \mathrm{~cm}^{-2}$ giving $\eta=87$.

In the framework of our approach, the restored spectrum may be considered as an average spectrum. This spectrum reveals features stemming from both the intergalactic incident radiation (depression between 3 and 4 Ryd in Fig. 6) and local processes in the cloud itself (enhanced re-emission of He II Ly $\alpha$, deep break at 4 Ryd). To reconstruct the true metagalactic spectrum, absorbers optically thin in He II are needed, i.e. those with $N(\mathrm{HI}) \lesssim 10^{15} \mathrm{~cm}^{-2}$.

\subsection{Absorption system at $Z_{\mathrm{abs}}=2.9659$ towards Q 0347-3819}

This system has been described in detail in Levshakov et al. (2003b). Since then Q 0347-3819 was re-observed with the VLT/UVES with a full wavelength coverage and longer 
Table 2. Physical parameters of the metal absorbers derived by the MCI procedure (limits are given at the $1 \sigma$ level).

\begin{tabular}{|c|c|c|c|c|}
\hline Parameter $^{a}$ & $\begin{array}{c}z_{\mathrm{abs}}=2.9171 \\
\mathrm{HE} 0940-1050 \\
\text { (2) }\end{array}$ & $\begin{array}{c}z_{\mathrm{abs}}=2.9659 \\
\mathrm{Q} 0347-3819 \\
\text { (3) }\end{array}$ & $\begin{array}{c}z_{\mathrm{abs}}=2.9375 \\
\mathrm{HE} 0940-1050 \\
\text { (4) }\end{array}$ & $\begin{array}{c}z_{\mathrm{abs}}=1.9426 \\
\mathrm{~J} 2233-606 \\
(5)\end{array}$ \\
\hline$U_{0}$ & $2.46 \mathrm{E}-2$ & $2.26 \mathrm{E}-2$ & $1.87 \mathrm{E}-1$ & $1.97 \mathrm{E}-2$ \\
\hline$N_{\mathrm{H}}, \mathrm{cm}^{-2}$ & $6.00 \mathrm{E} 20$ & $7.22 \mathrm{E} 17$ & 3.19E19 & $3.20 \mathrm{E} 19$ \\
\hline$\sigma_{\mathrm{v}}, \mathrm{km} \mathrm{s}^{-1}$ & 28.4 & 14.2 & 35.2 & 33.5 \\
\hline$\sigma_{\mathrm{y}}$ & 0.75 & 0.54 & 0.70 & 0.75 \\
\hline$Z_{\mathrm{C}}$ & $3.8 \mathrm{E}-7$ & $1.02 \mathrm{E}-4$ & $3.0 \mathrm{E}-6$ & $5.3 \mathrm{E}-5$ \\
\hline$Z_{N}$ & $\lesssim 1.3 \mathrm{E}-8$ & $9.0 \mathrm{E}-6$ & $\ldots$ & $4.9 \mathrm{E}-6$ \\
\hline$Z_{\mathrm{O}}$ & $\ldots$ & $\ldots$ & $1.2 \mathrm{E}-5$ & $<3.0 \mathrm{E}-4$ \\
\hline$Z_{\mathrm{Mg}}$ & $\ldots$ & $\cdots$ & $\ldots$ & $1.2 \mathrm{E}-5$ \\
\hline$Z_{\mathrm{Al}}$ & $\lesssim 3.8 \mathrm{E}-9$ & $\ldots$ & $\ldots$ & $8.0 \mathrm{E}-7$ \\
\hline$Z_{\mathrm{Si}}$ & $4.9 \mathrm{E}-8$ & $2.9 \mathrm{E}-5$ & $\ldots$ & $9.9 \mathrm{E}-6$ \\
\hline$\left[Z_{C}\right]$ & -2.8 & -0.38 & -1.90 & -0.66 \\
\hline$\left[Z_{N}\right]$ & $\lesssim-3.7$ & -0.82 & $\ldots$ & -1.1 \\
\hline$\left[Z_{\mathrm{O}}\right]$ & $\ldots$ & $\ldots$ & -1.57 & $<-0.2$ \\
\hline$\left[Z_{\mathrm{Mg}}\right]$ & $\ldots$ & $\ldots$ & $\ldots$ & -0.44 \\
\hline$\left[Z_{\mathrm{Al}}\right]$ & $\lessgtr-2.8$ & $\ldots$ & $\ldots$ & -0.52 \\
\hline$\left[Z_{\mathrm{Si}}\right]$ & -2.8 & -0.05 & $\ldots$ & -0.51 \\
\hline$N(\mathrm{H} \mathrm{I}), \mathrm{cm}^{-2}$ & $3.2 \mathrm{E} 17$ & 3.4E14 & $5.6 \mathrm{E} 14$ & $2.5 \mathrm{E} 16$ \\
\hline$N(\mathrm{C} \mathrm{II}), \mathrm{cm}^{-2}$ & $5.8 \mathrm{E} 12$ & $\leq 9.0 \mathrm{E} 11$ & $\ldots$ & $4.0 \mathrm{E} 13$ \\
\hline$N(\mathrm{Mg}$ II $), \mathrm{cm}^{-2}$ & $\ldots$ & $\ldots$ & $\ldots$ & 5.3E12 \\
\hline$N(\mathrm{Si}$ II $), \mathrm{cm}^{-2}$ & 8.9E11 & $\ldots$ & $\ldots$ & $5.6 \mathrm{E} 12$ \\
\hline$N(\mathrm{C}$ III $), \mathrm{cm}^{-2}$ & 2.0E14 & $4.8 \mathrm{E} 13$ & $5.7 \mathrm{E} 12$ & $1.0 \mathrm{E} 15$ \\
\hline$N(\mathrm{~N}$ III $), \mathrm{cm}^{-2}$ & $\lesssim 7.8 \mathrm{E} 12$ & 4.3E12 & $\ldots$ & $9.8 \mathrm{E} 13$ \\
\hline$N(\mathrm{Al} \mathrm{III}), \mathrm{cm}^{-2}$ & $\lesssim 2.2 \mathrm{E} 11$ & $\ldots$ & $\ldots$ & $1.5 \mathrm{E} 12$ \\
\hline$N(\mathrm{Si} \mathrm{III}), \mathrm{cm}^{-2}$ & $1.2 \mathrm{E} 13$ & $3.8 \mathrm{E} 12$ & $\ldots$ & 7.9E13 \\
\hline$N(\mathrm{C}$ IV $), \mathrm{cm}^{-2}$ & $2.4 \mathrm{E} 13$ & $1.8 \mathrm{E} 13$ & $9.2 \mathrm{E} 12$ & 3.9E14 \\
\hline$N(\mathrm{Si}$ IV $), \mathrm{cm}^{-2}$ & $7.6 \mathrm{E} 12$ & $6.0 \mathrm{E} 12$ & $\ldots$ & $5.8 \mathrm{E} 13$ \\
\hline$N(\mathrm{~N} \mathrm{v}), \mathrm{cm}^{-2}$ & $\ldots$ & $\ldots$ & $\ldots$ & $9.2 \mathrm{E} 12$ \\
\hline$N(\mathrm{O} \mathrm{VI}), \mathrm{cm}^{-2}$ & $\ldots$ & $\ldots$ & $4.2 \mathrm{E} 13$ & $<1.4 \mathrm{E} 14$ \\
\hline$\langle T\rangle, \mathrm{K}$ & $2.8 \mathrm{E} 4$ & $1.3 \mathrm{E} 4$ & $3.7 \mathrm{E} 4$ & $1.8 \mathrm{E} 4$ \\
\hline$N(\mathrm{He} \mathrm{II})^{b}, \mathrm{~cm}^{-2}$ & $2.8 \mathrm{E} 19$ & $2.1 \mathrm{E} 16$ & $8.2 \mathrm{E} 16$ & $1.1 \mathrm{E} 18$ \\
\hline$\eta^{b}$ & 87 & 62 & 146 & 44 \\
\hline
\end{tabular}

${ }^{a} Z_{\mathrm{X}}=N_{\mathrm{X}} / N_{\mathrm{H}} ;\left[Z_{\mathrm{X}}\right]=\log \left(N_{\mathrm{X}} / N_{\mathrm{H}}\right)-\log \left(N_{\mathrm{X}} / N_{\mathrm{H}}\right)_{\odot}$.

${ }^{b}$ Predicted values.

exposure time. This resulted in a high-quality spectrum with a resolution of $\sim 6 \mathrm{~km} \mathrm{~s}^{-1}$ and $S / N=50-100$. Here we repeat the analysis for the new data.

The hydrogen and metal absorption lines of the $z_{\text {abs }}=2.9659$ system are shown in Fig. 7 (histograms). Neither C II $\lambda 1334 \AA$ (undetected) nor Si II $\lambda 1260 \AA$ (blended) can be used. This makes the presence of the C III $\lambda 977 \AA$ line crucial for the SED estimation since Si III, Si IV and C IV lines can be fitted with a very broad range of ionizing spectra (constrained only by the condition $[\mathrm{Si} / \mathrm{C}]<0.5$ ). Although we did not find any metal line candidate for blending with C III, we cannot exclude blending with $\operatorname{Ly} \alpha$ forest absorption. Thus, the analysis below should be taken with caution and it is valid only if the absorption at the position of C III is entirely due to this ion.

Calculations with the HM ionizing background showed that this spectrum significantly underestimates C III. Our trials to reconstruct the underlying continuum shape were conducted with two sets of factors, with and without accounting for the He II Ly $\alpha$ absorption. In both cases it was possible to obtain 


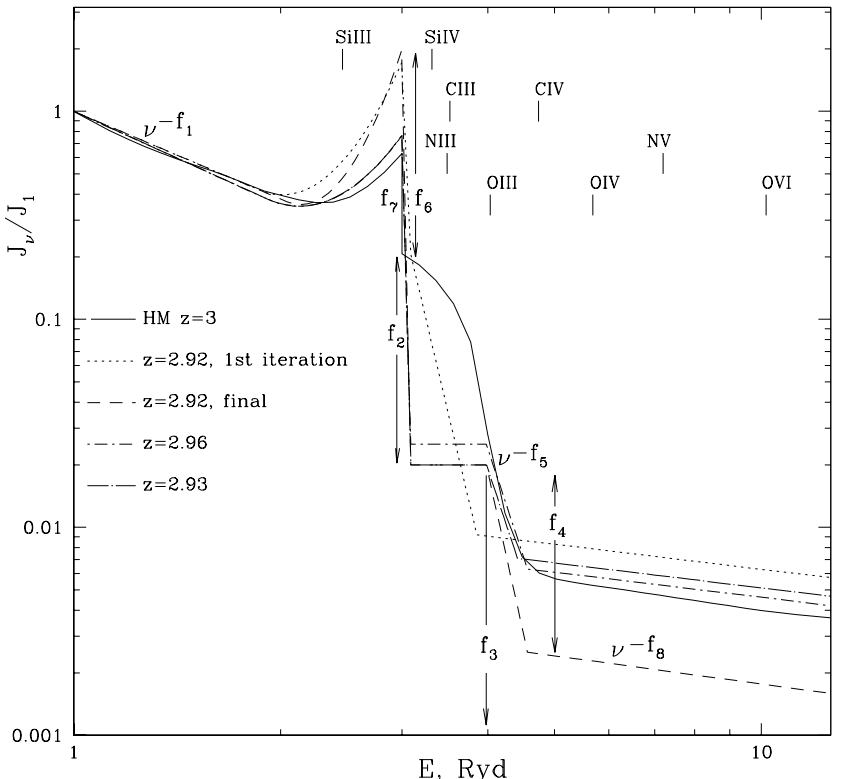

Fig. 6. SEDs recovered from the absorption line systems. The positions of ionization thresholds of different ions are indicated by tick marks. The definition of factors $\left\{f_{i}\right\}$ are given in Sect. 3.1. For details, see text.

the appropriate ionizing spectra. However, the spectrum estimated without He II Ly $\alpha$ absorption shows significantly enhanced emission at $3 \mathrm{Ryd}$ and at the same time is harder at $E>4$ Ryd compared to the initial HM spectrum. Since the absorber under study has $N(\mathrm{HI})=3.4 \times 10^{14} \mathrm{~cm}^{-2}$ and is optically thin in He II, this type of ionizing spectrum is obviously an artifact and should be rejected.

The recovered spectrum with the intensity depression at 3 Ryd $<\mathrm{E}<4$ Ryd is shown in Fig. 6 by the point-dashed line with the corresponding synthetic profiles plotted by the smooth lines in Fig. 7 (physical parameters are given in Table 2, Col. 3). Since the depth of the He II Ly $\alpha$ trough (factor $f_{2}$ ) quite strongly affects the ion fractions, its value is estimated with an accuracy better than 0.1 dex. The depth of the He II continuum absorption (factor $f_{4}$ ) has an uncertainty of about 0.15 dex. The predicted He II column density is $2.1 \times 10^{16} \mathrm{~cm}^{-2} \quad(\eta=62)$ and, hence, this cloud is optically thin in He II, $\tau_{\text {He II }}^{\text {cont }} \simeq 0.03$. Thus, the recovered background coincides in this case with the infalling radiation spectrum and is not modified by local effects. From the depth of the He II Ly $\alpha$ trough we can estimate the opacity of the intergalactic He II Ly $\alpha$ absorption as $\tau_{\mathrm{He} \text { II }}=2.1 \pm 0.2$.

\subsection{Absorption system at $z_{\mathrm{abs}}=2.9375$ towards HE 0940-1050}

The absorption system at $z_{\mathrm{abs}}=2.9375$ towards HE 0940-1050 reveals metal lines of C III $\lambda 977 \AA$, C IV $\lambda \lambda 1548,1550 \AA$ and O VI $\lambda 1037 \AA$ (OVI $\lambda 1031 \AA$ is blended, as well as N V $\lambda \lambda 1238,1242 \AA$ ) (Fig. 8). We cannot exclude that the observed O VI $\lambda 1037 \AA$ and C III lines are contaminated by some hydrogen absorption. However, no metal line candidates for blending were found. As in the case of the $z_{\mathrm{abs}}=2.9659$ system described in the preceding subsection, the analysis below is valid only if the intensities are entirely due to absorption of the corresponding ions.

Two lines of one element along with one line of another can be described with a very broad range of ionizing spectra. However, different spectra will deliver different abundance ratios $[\mathrm{O} / \mathrm{C}]$ and this can be used to constrain the allowable SEDs. Measurements of $[\mathrm{O} / \mathrm{C}]$ in Galactic and extragalactic H II regions and in metal-poor halo stars indicate that a safe upper bound for [O/C] is 0.5 (Henry et al. 2000; Akerman et al. 2004).

Calculations with the HM spectrum produce for this absorption system $[\mathrm{O} / \mathrm{C}]>1$, suggesting that the shape of the input ionizing background is inadequate. To meet $[\mathrm{O} / \mathrm{C}]<0.5$, the ionizing spectrum should be either significantly harder (by $0.5 \mathrm{dex})$ at $E>4$ Ryd or should have a step-like depression at $E \gtrsim 3 \mathrm{Ryd}$. The second option is preferable since this step is also present in the spectrum recovered from the absorber at $z_{\mathrm{abs}}=2.9171$ along the same line of sight (Sect. 3.1). The step at 3 Ryd $<E<4$ Ryd strongly affects the $\mathrm{C}$ III/C IV ratio leading to the result that the observed ratio is obtained at a higher ionization parameter compared to the HM spectrum and, hence, the corresponding O VI fraction is larger.

Using the HM spectrum as an initial approximation and varying the depth of the He II Ly $\alpha$ absorption trough (i.e. one-factor experiment) we adjusted the spectral shape in a way that enables the description of the observed lines with $[\mathrm{O} / \mathrm{C}]<0.5$. The obtained spectral shape is shown in Fig. 6 (long-dashed line), with the synthetic profiles plotted by the smooth lines in Fig. 7 and the physical parameters given in Table 2, Col. 4. The uncertainty in the step depth is of $0.1 \mathrm{dex}$ (given the energy above 4 Ryd at the level of the HM spectrum). The predicted column density of the singly ionized he$\operatorname{lium} N(\mathrm{He}$ II $)=8.2 \times 10^{16} \mathrm{~cm}^{-2}$ translates to $\eta=146$ which is almost 2.5 times higher than in the $z_{\mathrm{abs}}=2.9659$ system.

\subsection{Absorption system at $Z_{\mathrm{abs}}=1.9426$ towards J 2233-606}

This system has been described by Prochaska \& Burles (1999), D'Odorico \& Petitjean (2001) and Levshakov et al. (2002). A wide wavelength coverage provided by combining the VLT/UVES and HST/STIS spectra allows us to identify many higher-order hydrogen lines and numerous metal transitions (Fig. 9). Unfortunately, the important C II $\lambda 1334 \AA$ line is blended with Ly- $\alpha$ forest absorption, and C II $\lambda 1036 \AA$ is very noisy, but this system is nevertheless worth investigating due to the rare occurrence of the simultaneous presence of low- (Si II, $\mathrm{Mg}$ II) and high ionization lines ( $\mathrm{N} \mathrm{V}$.

Calculations with the $z=2 \mathrm{HM}$ ionizing spectrum (Fig. 10, solid line) describe most of the observed line profiles except N III $\lambda 989 \AA$ and N v $\lambda \lambda 1238,1242 \AA$ which are over- and underestimated, respectively. The SED was adjusted in two experiments with factor sets both accounting for the Gunn-Peterson (GP) absorption and without it, and with the response maximizing the product of the ratios $\Upsilon_{\mathrm{NV}} / \Upsilon_{\mathrm{N} \text { III }}, \Upsilon_{\mathrm{CII}} / \Upsilon_{\mathrm{CIV}}$, and $\Upsilon_{\text {Si IV }} / \Upsilon_{\text {Si III. All trials showed that to reproduce the nitrogen }}$ lines the ionizing background should be significantly harder 


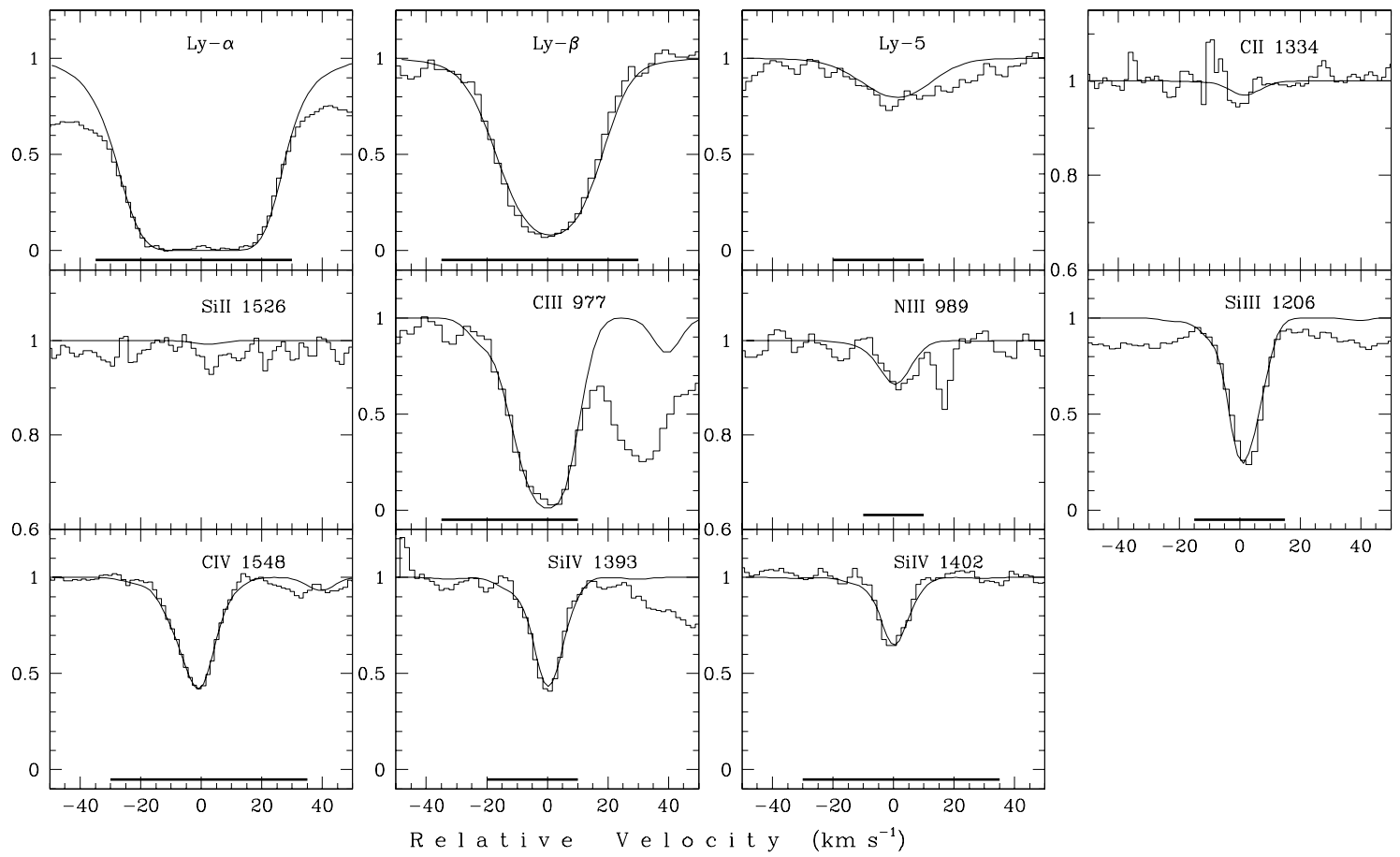

Fig. 7. Hydrogen and metal absorption lines associated with the $z_{\mathrm{abs}}=2.9659$ system towards Q 0347-3819 (histograms). Synthetic profiles corresponding to the MCI solution for the final adjusted SED (dot-short-dashed line in Fig. 6) are shown by the smooth curves. The corresponding physical parameters are listed in Table 2, Col. 3. Bold horizontal lines mark pixels included in the optimization procedure. The zero radial velocity is fixed at $z=2.9659$.

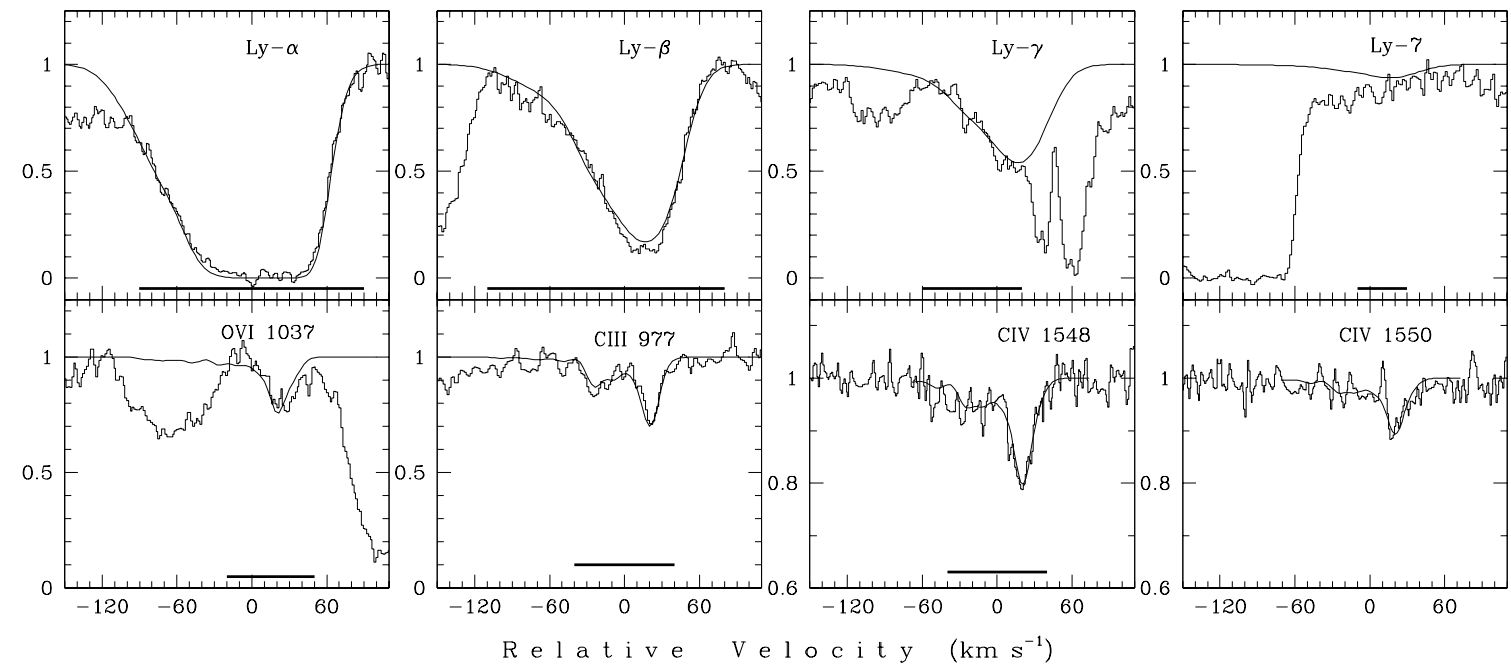

Fig. 8. Same as Fig. 7 but for the $z_{\text {abs }}=2.9375$ system towards HE 0940-1050. The MCI synthetic profiles (smooth curves) are calculated with the final adjusted SED shown by the dot-long dashed line in Fig. 6. The corresponding physical parameters are listed in Table 2, Col. 4. The zero radial velocity is fixed at $z=2.9375$.

at $E>4$ Ryd than the initial HM spectrum. Unfortunately, because of the low quality of the C II $\lambda \lambda 1334,1036 \AA$ lines, it was impossible to distinguish between the ionizing spectra with or without the GP absorption. In fact, both recovered SEDs (shown by the dotted and dashed lines in Fig. 10) give identical fitting to the data. The synthetic profiles are shown by the smooth lines in Fig. 9. However, an upper limit can be set for a putative GP He II absorption at $z=2: \tau_{\mathrm{He} \mathrm{II}}<1.8$.
The predicted column density of He II of $1.1 \times 10^{18} \mathrm{~cm}^{-2}$ $(\eta=44)$ gives the optical depth in the He II continuum of 1.5 . In principle, such optical depth can soften noticeably the incident ionizing continuum at $E>4$ Ryd. However, the observed lines of $\mathrm{N} \mathrm{III} \mathrm{and} \mathrm{N}$ V clearly require a hard spectrum in this energy range. This contradiction can be explained in two ways: either the $\mathrm{N} v$ lines arise in the external regions of the absorbing cloud where the incident ionizing continuum is not yet 


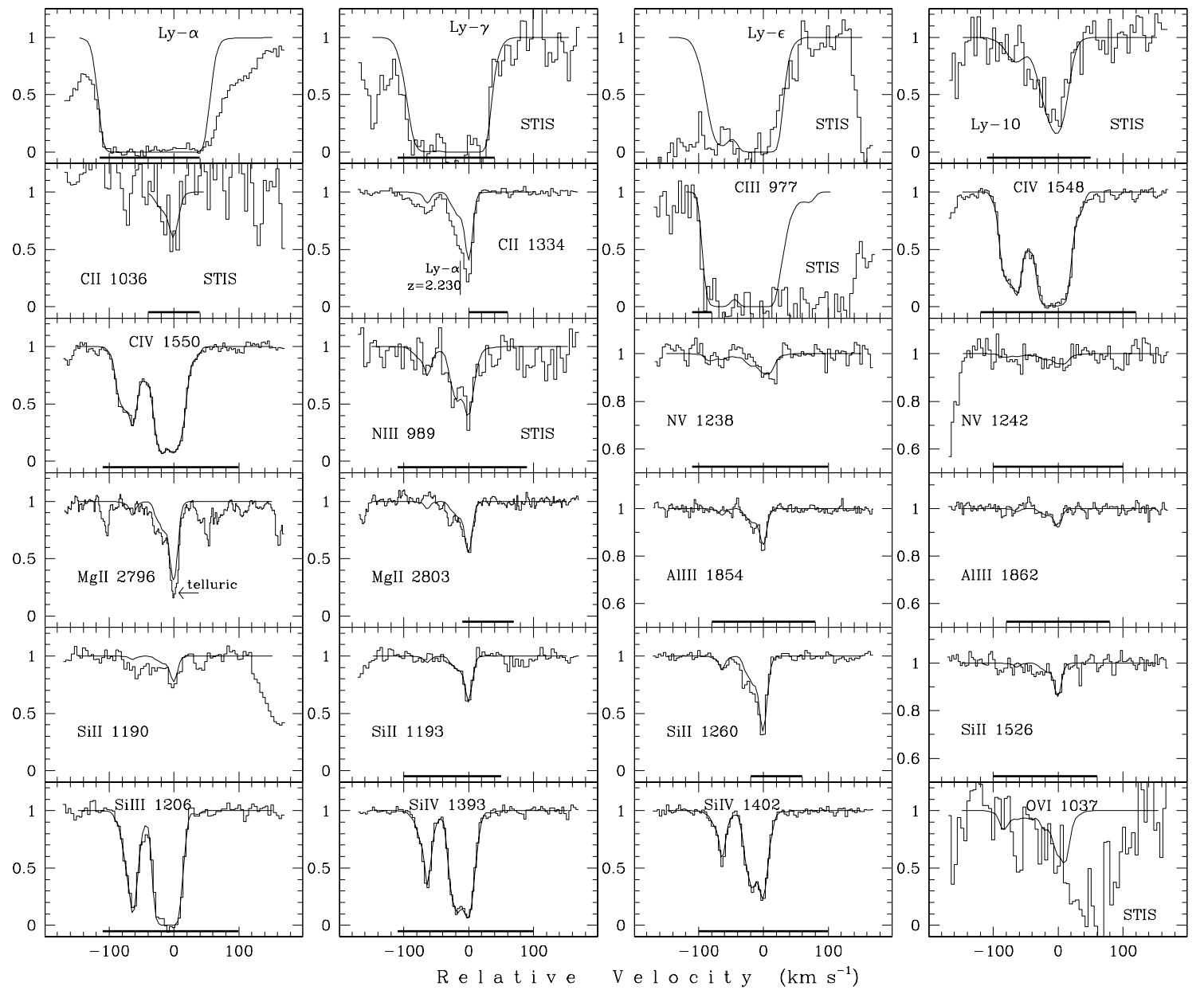

Fig. 9. Same as Fig. 7 but for the $z_{\text {abs }}=1.9426$ system towards J 2233-606. The MCI synthetic profiles (smooth curves) are calculated with the SEDs shown by the dotted and dashed lines in Fig. 10. The corresponding physical parameters are listed in Table 2, Col. 5. The zero radial velocity is fixed at $z=1.9426$.

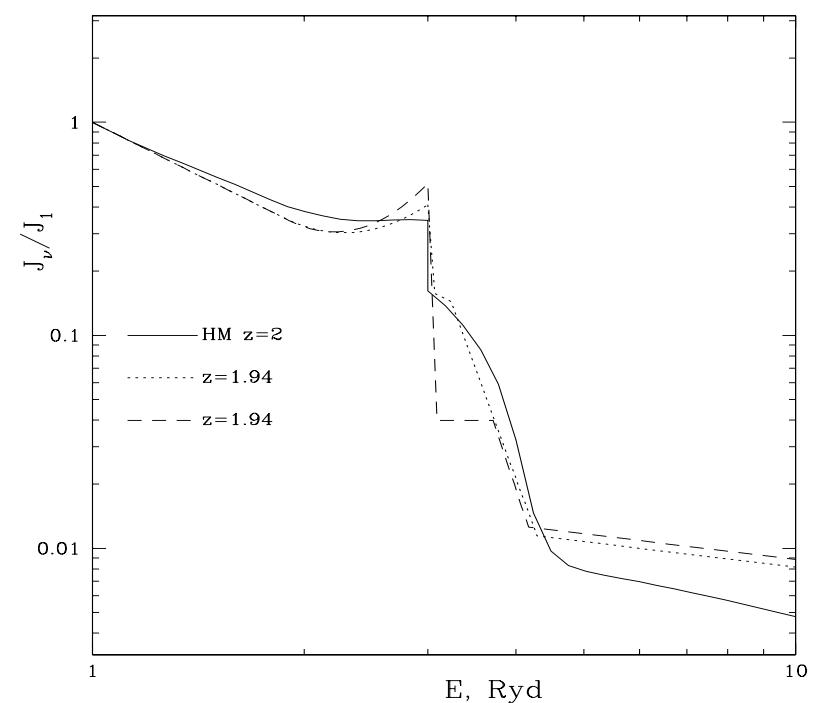

Fig. 10. SEDs recovered from the absorption line system at $z=1.9426$ towards J 2233-606. For details, see text.

distorted by the He II continuum absorption, or the density variations along the line of sight make the effective optical depth smaller. In both cases we can conclude that the metagalactic ionizing spectrum at $z \sim 2$ is harder at $E>4$ Ryd as compared to the spectrum predicted by HM.

\section{Conclusions}

We have proposed a method to estimate the shape of the ionizing continuum from metal lines observed in the intervening absorption systems. The implementation includes the following steps:

- parameterization of the spectral shape by means of a set of factors based on physical processes relevant for the IGM;

- choice of some quantitative measure (response) to estimate the fitness of a trial shape;

- generation of trial shapes by randomization of the factor values according to some experimental design;

- evaluation of the factor effects;

- estimation of the optimal shape by moving in factor space towards the ascending fitness.

The result depends on the number of metal lines involved in the analysis: in general, the more lines of different ionic transitions of different elements detected in an absorption system, the higher is the accuracy of the recovered spectral shape. In some 
cases additional information concerning, for instance, element abundance ratios can significantly constrain the factor values, in particular when only a few metal lines are available.

Although the main objective of this work is to illustrate how the proposed approach can be used in practice, some physical results are worth mentioning:

1. The metagalactic ionizing spectrum at redshift $z \sim 3$ has breaks at 3 and 4 Ryd and is quite hard at $E>4 \mathrm{Ryd}$, at least as hard as the model metagalactic spectrum of Haardt \& Madau (1996).

2. The intensity decrease between 3 and 4 Ryd is probably produced mostly by He II Ly $\alpha$ absorption in the intergalactic diffuse gas since line-blanketing from discrete Ly $\alpha$ forest clouds cannot account for significant He II opacity with such a hard ionizing spectrum (Fardal et al. 1998; Zheng et al. 1998). Thus, the intensity depression at $3 \mathrm{Ryd}<E<$ 4 Ryd in the spectrum of the metagalactic ionizing radiation may be an imprint of a true He II Gunn-Peterson effect.

3. The He II opacity estimated from the depth of the He II Ly $\alpha$ absorption trough is $\tau_{\mathrm{He} \text { II }} \sim 2$. This should be considered as an average optical depth since in our approach the He II Ly $\alpha$ absorption is approximated by a straight step. As known from observations, He II opacity at $z \sim 3$ fluctuates revealing both absorption troughs and opacity gaps. For reference, $\tau_{\text {He II }} \sim 4$ is measured in absorption troughs at $2.77<z<2.87$ in the spectrum of HE 2347-4342 (Zheng et al. 2004) along with $\tau_{\text {He II }}<0.5$ in the opacity gaps at $z_{\mathrm{abs}}=2.817$ and 2.866 (Reimers et al. 1997).

4. A putative galactic contribution to the ionizing background spectrum (e.g. Pettini et al. 2001; Ciardi et al. 2002; Fujita et al. 2003), if present, would significantly soften the spectrum at $E>4$ Ryd. We do not find any traces of soft components in the recovered spectra: even in the absorption systems at $z=2.96$ and $z=1.94$, where high metallicity supposes their kinship with galaxies, the observed line intensities point to a hard ionizing background. Thus, we can conclude that the ionizing background at $z \sim 3$ and $z \sim 2$ is dominated by QSOs.

Acknowledgements. The work of I.I.A. and S.A.L. is supported by the RFBR grant 03-02-17522 and by the RLSS grant 1115.2003.2.

\section{Appendix A: An example of experimental design for 7 factors}

The factors are tested at two levels: $f_{i}=f_{0, i} \pm \sigma_{i}$, where $\sigma_{i}$ is a suitable scale. Usually $\sigma_{i} \sim(0.1-0.2) f_{0, i}$, in order to maintain the linear dependence of the response $\tilde{\mathcal{R}}$ on the factors. The normalized and centered factor values at the upper and lower levels are, respectively, +1 and -1 . Then a 7 -factorial saturated simplex design can be defined by the following symmetric and orthogonal matrix $\left\{\hat{f}_{i j}\right\}$ (Nalimov 1971):

\begin{tabular}{lccccccc|c}
\hline \hline & $\hat{f}_{1}$ & $\hat{f}_{2}$ & $\hat{f}_{3}$ & $\hat{f}_{4}$ & $\hat{f}_{5}$ & $\hat{f}_{6}$ & $\hat{f}_{7}$ & $\tilde{\mathcal{R}}$ \\
\hline+1 & -1 & -1 & -1 & +1 & +1 & +1 & -1 & $\mathcal{R}_{1}$ \\
+1 & +1 & -1 & -1 & -1 & -1 & +1 & +1 & $\mathcal{R}_{2}$ \\
+1 & -1 & +1 & -1 & -1 & +1 & -1 & +1 & $\mathcal{R}_{3}$ \\
+1 & +1 & +1 & -1 & +1 & -1 & -1 & -1 & $\mathcal{R}_{4}$ \\
+1 & -1 & -1 & +1 & +1 & -1 & -1 & +1 & $\mathcal{R}_{5}$ \\
+1 & +1 & -1 & +1 & -1 & +1 & -1 & -1 & $\mathcal{R}_{6}$ \\
+1 & -1 & +1 & +1 & -1 & -1 & +1 & -1 & $\mathcal{R}_{7}$ \\
+1 & +1 & +1 & +1 & +1 & +1 & +1 & +1 & $\mathcal{R}_{8}$ \\
\hline
\end{tabular}

The first column is added to estimate the free term $\beta$ in the model (1). Such an experimental design is called a "two level saturated plan" since here all degrees of freedom $N=8$ are used to estimate 8 regression coefficients $\left(\left\{\alpha_{i}\right\}_{i=1}^{7}\right.$ and $\left.\beta\right)$ :

$\alpha_{i}=\frac{1}{N} \sum_{j=1}^{N} \hat{f}_{j i} \mathcal{R}_{j}$,

and

$\beta=\frac{1}{N} \sum_{j=1}^{N} \mathcal{R}_{j}$.

Other plans with different numbers of levels and treatments (rows in the matrix) can be used as well.

After the regression coefficients for the model (1) are found, new factor values can be calculated from

$\hat{f}_{i}^{\text {new }}=f_{0, i}+\Delta \mathcal{R} \sigma_{i} \mu_{i}$,

where $\mu_{i}=\alpha_{i} / \sqrt{\sum_{j=1}^{7} \alpha_{j}^{2}}$ are direction cosines and $\Delta \mathcal{R}$ is the increment of the response. The value of $\Delta \mathcal{R}$ is restricted by the condition $\chi^{2} \sim 1$ for each absorption line (or its portion) involved in the optimization procedure.

\section{References}

Akerman, C. J., Carigi, L., Nissen, P. E., Pettini, M., \& Asplund, M. 2004, A\&A, 414, 931

Anderson, S. F., Hogan, C. J., Williams, B. F., \& Carswell, R. F. 1999, AJ, 117, 56

Asplund, M., Grevesse, N., \& Sauval, A. J. 2005, in Cosmic Abundances as Records of Stellar Evolution and Nucleosynthesis, ed. F. N. Bash, \& T. G. Barnes, ASP Conf. Ser., in press [arXiv: astro-ph/0410214]

Bergeron, J., \& Stasinska, G. 1986, A\&A, 169, 1

Box, G. E. P., Hunter, W. G., \& Hunter, J. S. 1978, Statistics for Experimenters: An Introduction to Design, Data Analysis, and Model Building (NY: Wiley \& Sons)

Chaffee, F. H., Jr., Foltz, C. B., Bechtold, J., \& Weymann, R. J. 1986, ApJ, 301, 116 
Ciardi, B., Bianchi, S., \& Ferrara, A. 2002, MNRAS, 331, 463

D’Odorico, V., \& Petitjean, P. 2001, A\&A, 370, 729

Fardal, M. A., Giroux, M. L., \& Shull, M. 1998, AJ, 115, 2206

Ferland, G. J. 1997, A Brief Introduction to Cloudy, Internal Rep., Lexington, Univ. Kentucky

Fujita, A., Martin, C. L., MacLow, M., \& Abel, T. 2003, ApJ, 599, 50

Giroux, M. L., \& Shull, J. M. 1997, AJ, 113, 1505

Haardt, F., \& Madau, P. 1996, ApJ, 461, 20 [HM]

Heap, S. R., Williger, G. M., Smette, A., et al. 2000, ApJ, 534, 69

Henry, R. B. C., Edmunds, M. G., \& Köppen, J. 2000, ApJ, 541, 660

Jakobsen, P., Jansen, R. A., Wagner, S., \& Reimers, D. 2003, A\&A, 397,891

Levshakov, S. A., Agafonova, I. I., \& Kegel, W. H. 2000, A\&A, 360, 833 [LAK]

Levshakov, S. A., Agafonova, I. I., Centurión, M., \& Mazets, I. E. 2002, A\&A, 383, 813

Levshakov, S. A., Agafonova, I. I., Reimers, D., \& Baade, R. 2003a, A\&A, 404, 449
Levshakov, S. A., Agafonova, I. I., D’Odorico, S., Wolfe, A. M., \& Dessauges-Zavadsky, M. 2003b, ApJ, 582, 596

Levshakov, S. A., Agafonova, I. I., Centurión, M., \& Molaro, P. 2003c, A\&A, 397, 851

Mathews, W. G., \& Ferland, G. J. 1987, ApJ, 323, 456 [MF]

Morton, D. C. 2003, ApJS, 149, 205

Nalimov, V. V. 1971, The theory of experiment (Moscow: Nauka)

Prochaska, J. K., \& Burles, S. M. 1999, AJ, 117, 1957

Reimers, D., Köhler, S., Wisotzki, L., et al. 1997, A\&A, 327, 890

Pettini, M., Shapley, A. E., Steidel, C. C., et al. 2001, ApJ, 554, 981

Shull, J. M., Tumlinson, J., Giroux, M. L., Kriss, G. A., \& Reimers, D. 2004, ApJ, 600,570

Smette, A., Heap, S. R., Williger, G. M., et al. 2002, ApJ, 564, 542

Vogel, S., \& Reimers, D. 1995, A\&A, 294, 377

Zheng, W., Davidsen, A. F., \& Kriss, G. A. 1998, AJ, 115, 391

Zheng, W., Chiu, K., Anderson, S. F., et al. 2004a, AJ, 127, 656

Zheng, W., Kriss, G. A., Deharveng, J.-M., et al. 2004b, ApJ, 605, 631 